\title{
Cognitive Best Worst Method for Multiattribute Decision-Making
}

\author{
Hongjun Zhang, Chengxiang Yin, Xiuli Qi, Rui Zhang, and Xingdang Kang \\ PLA University of Science and Technology, No. 1, Haifu Lane, Qinhuai District, Nanjing City, Jiangsu Province, China \\ Correspondence should be addressed to Chengxiang Yin; 425850693@qq.com
}

Received 14 November 2016; Revised 20 March 2017; Accepted 21 March 2017; Published 4 April 2017

Academic Editor: Anna M. Gil-Lafuente

Copyright (c) 2017 Hongjun Zhang et al. This is an open access article distributed under the Creative Commons Attribution License, which permits unrestricted use, distribution, and reproduction in any medium, provided the original work is properly cited.

\begin{abstract}
Pairwise comparison based multiattribute decision-making (MADM) methods are widely used and studied in recent years. However, the perception and cognition towards the semantic representation for the linguistic rating scale and the way in which the pairwise comparisons are executed are still open to discuss. The commonly used ratio scale is likely to produce misapplications and the matrix based comparison style needs too many comparisons and is not able to guarantee the consistency of the matrix when the number of objects involved is large. This research proposes a new MADM method CBWM (Cognitive Best Worst Method) which adopts interval scale to represent the pairwise difference and only compares each object to the best object and the worst object rather than all the other objects. CBWM is a vector based method which only needs $2 n-3$ pairwise comparisons and is more likely to generate consistent comparisons and reliable results. The theoretical analysis and a real world application demonstrate the effectiveness of CBWM.
\end{abstract}

\section{Introduction}

A typical multiattribute decision-making (MADM) problem can be described in Table 1. In Table 1, $A=\left\{a_{1}, a_{2}, \ldots, a_{m}\right\}$ is a set of alternatives (actions, stimuli), $G=\left\{g_{1}, g_{2}, \ldots, g_{n}\right\}$ is a set of criteria, and $g_{i j}$ is the performance of alternative $a_{i}$ with respect to criterion $g_{j}$. There are four kinds of tasks in MADM: description $(P \cdot \delta)$, choice $(P \cdot \alpha)$, sorting $(P$. $\beta)$, and ranking $(P \cdot \gamma)[1] . P \cdot \delta$ elaborates the alternatives (building family of criteria, determining performances, weights, thresholds, etc.), $P \cdot \alpha$ aims at selecting a small number (as small as possible) of good alternatives, $P \cdot \beta$ assigns each alternative into a family of predefined categories, and $P \cdot \gamma$ generates a partial or complete ordering for the alternatives. To handle MADM problems, various methods such as multiattribute utility theory (UTA [2], UTAGMS [3], AHP [4], ANP [5], MACBETH [6], P-CNP [7], etc.), outranking methods (ELECTRE [8], PROMETHEE [9], etc.), and some nonclassical methods (DRSA [10], FAHP [11], FCNP [12], etc.) have been proposed. To get a whole picture of these methods, we refer to [1,13-18].

Among these methods, AHP has been increasingly studied by plenty of researchers and applied in various applications due to its simplicity and practicability. AHP is based on pairwise comparison which is first introduced by Thurstone [19] and derives priority vector from the comparison matrix $P=\left\{p_{i j}\right\}$ whose entry $p_{i j}$ indicates the relative importance of object $i$ dominating object $j$. In AHP, the paired difference is measured by the ratio scale and correspondingly $P$ is a pairwise reciprocal matrix (PRM).

Yuen $[7,20-22]$ pointed out that the paired ratio scale inappropriately represents the human perception or cognition of paired difference and potentially producing misapplications. Taking the comparison of two persons height, for example, if $A$ is $1.4 \mathrm{~m}$ and $B$ is $1.5 \mathrm{~m}$, then we may say that B is slightly taller than A. However, if ratio scale is applied, the comparison result will be 2; for example, B is two times taller than A which is obviously unreasonable. The exaggeration of difference may cause the rank reversal problem and mislead the decision-maker. To handle this problem, Yuen proposed replacing the ratio scale by interval or differential scale which measures the difference rather than the quotient of the importance of two objects and accordingly converts the pairwise reciprocal matrix (PRM) to the pairwise opposite matrix $(\mathrm{POM})$ and then derives the priority vector from POM by Primitive Cognitive Network Process (P-CNP). 
TABLE 1: Description of MADM problem.

\begin{tabular}{lcccc}
\hline & $g_{1}$ & $g_{2}$ & $\cdots$ & $g_{n}$ \\
\hline$a_{1}$ & $g_{11}$ & $g_{12}$ & $\cdots$ & $g_{1 n}$ \\
$a_{2}$ & $g_{21}$ & $g_{22}$ & $\ldots$ & $g_{2 n}$ \\
$\vdots$ & $\vdots$ & $\vdots$ & $\ddots$ & $\vdots$ \\
$a_{m}$ & $g_{m 1}$ & $g_{m 2}$ & $\ldots$ & $g_{m n}$ \\
\hline
\end{tabular}

Apart from the scale problem, Rezaei [23, 24] pointed out that the unstructured way in which pairwise comparisons are executed would cause the inconsistency of the pairwise comparison matrix. In order to improve the consistency, Rezaei proposed the Best Worst Method (BWM) which only compares each criterion to the best (most important, most desirable) and the worst (least important, least desirable) criterion, respectively. The pairwise comparison results are two vectors based on which a nonlinear model and a linear model are designed to derive the priority vector. BWM not only leads to more consistent comparisons but also reduces the number of comparisons.

Both P-CNP and BWM have been proved to be effective and been applied in some real world applications [22, 25-34]. However, to the best of our knowledge, there has not been a pairwise comparison based MADM method that has the two aspects of advantages possessed by P-CNP and BWM at the same time. In this paper, we propose the Cognitive Best Worst Method (CBWM) which executes the pairwise comparisons in the same way as BWM and adopts the interval scale to measure the difference. CBWM possesses the advantages of both P-CNP and BWM and shows better characteristics than both the two methods. Compared to P-CNP, CBWM needs less pairwise comparisons and is likely to get more consistent pairwise comparisons. Compared to BWM, CBWM adopts the interval scale which is more suitable for measuring the cognitive paired comparisons and acquires more reliable results. The theoretical and experimental analyses demonstrate the good performance of CBWM.

The rest of this paper is organized as follows. Section 2 reviews P-CNP and BWM. Section 3 illustrates the Cognitive Best Worst Method. Section 4 presents an application using CBWM and compares CBWM to AHP, P-CNP, and BWM. Section 5 concludes the research.

\section{Review of P-CNP and BWM}

In this section, we briefly review the concept and process of P-CNP and BWM. For the detailed description of the two methods, we refer to [7] and [23, 24], respectively.

2.1. $P$-CNP. The P-CNP is the cognitive architecture which comprises cognitive decision processes: Problem Cognitive Process (PCP), Cognitive Assessment Process (CAP), Cognitive Prioritization Process (CPP), Multiple Information Fusion Process (MIP), and Decision Volition Process (DVP).

2.1.1. PCP. In PCP, the goal of the decision-maker and the criteria set $G=\left\{g_{1}, g_{2}, \ldots, g_{n}\right\}$ and the alternatives set
$A=\left\{a_{1}, a_{2}, \ldots, a_{m}\right\}$ are determined. Besides the elements aforementioned, a measurement scale schema $(\mathcal{N}, \bar{X})$ which maps the verbal scales $\mathcal{N}$ to the corresponding numerical scales $\bar{X}$ is also needed. As P-CNP adopts interval scales to measure the pairwise differences, the numerical representations should be in the following form:

$$
\bar{X}=\left\{\alpha_{i}=\frac{i \kappa}{\tau} \mid \forall i \in\{-\tau, \ldots,-1,0,1, \ldots, \tau\}, \kappa>0\right\} .
$$

$\kappa$ is the normal utility which is the mean of individual utility values of the comparison objects. $2 \tau+1$ is the number of intervals in the scale schema. From the definition of $\bar{X}$ we can get $\max (\bar{X})=\kappa$. For the convenience of computation, we set $\kappa=\tau$; then each element in $\bar{X}$ will be an integer. Table 2 gives an example of the ratio scale and the interval scale $(\tau=\kappa=8)$.

2.1.2. CAP. In CAP, the pairwise comparisons are executed and the pairwise opposite matrix (POM) is constructed. Let an ideal utility vector of the objects involved in the pairwise comparisons be $V=\left(v_{1}, v_{2}, \ldots, v_{n}\right)$; then the ideal POM will be $\widetilde{B}=\left\{\widetilde{b}_{i j}\right\}$, where $\widetilde{b}_{i j}=v_{i}-v_{j}$. Let $B=\left[b_{i j}\right]$ be the subjective judgment POM provided by the experts; then $\widetilde{B}$ is determined by $B$ in the following way:

$$
\begin{aligned}
\widetilde{B} & =\left\{\widetilde{b}_{i j}\right\}=\left[\begin{array}{cccc}
0 & v_{1}-v_{2} & \cdots & v_{1}-v_{n} \\
v_{2}-v_{1} & 0 & \cdots & v_{2}-v_{n} \\
\vdots & \vdots & \ddots & \vdots \\
v_{n}-v_{1} & v_{n}-v_{2} & \cdots & 0
\end{array}\right] \\
& \simeq\left[\begin{array}{cccc}
0 & b_{12} & \cdots & b_{1 n} \\
b_{21} & 0 & \cdots & b_{2 n} \\
\vdots & \vdots & \ddots & \vdots \\
b_{n 1} & b_{n 2} & \cdots & 0
\end{array}\right]=\left[b_{i j}\right]=B .
\end{aligned}
$$

The pairwise comparison matrix is called POM because $b_{i j}=-b_{j i}$ holds for all $i, j \in\{1,2, \ldots, n\}$. In [21], Yuen proposed that a POM $B=\left[b_{i j}\right]$ is perfectly accordant if

$$
\begin{aligned}
b_{i k}+b_{k j} & =b_{i j}, \quad \forall i, j, k \in\{1,2, \ldots, n\}, \\
\text { or } b_{i k}-b_{j k} & =b_{i j}, \quad \forall i, j, k \in\{1,2, \ldots, n\} .
\end{aligned}
$$

The accordance index (AI) is used to measure the degree of accordance.

$$
\mathrm{AI}=\frac{1}{n^{2}} \sum_{i=1}^{n} \sum_{j=1}^{n} \sqrt{\frac{1}{n} \sum_{k=1}^{n}\left(\frac{1}{\kappa}\left(b_{i k}+b_{k j}-b_{i j}\right)\right)^{2}} .
$$

If $\mathrm{AI}=0$, then $B$ is perfectly accordant; if $0<\mathrm{AI} \leq 0.1$, then $B$ is satisfactory; if $\mathrm{AI}>0.1, B$ is unsatisfactory.

2.1.3. CPP. The purpose of CPP is to derive the priority vector from the POM. The two common used methods are primitive 
TABLE 2: Scale schemas: ratio scales and interval scales.

\begin{tabular}{lccc}
\hline$i$ & Verbal scales & Ratio scales & Interval scales \\
\hline 0 & Equally & 1 & 0 \\
1 & Weakly & 2 & 1 \\
2 & Moderately & 3 & 2 \\
3 & Moderately plus & 4 & 3 \\
4 & Strongly & 5 & 5 \\
5 & Strongly plus & 6 & 6 \\
6 & Very strongly & 7 & 7 \\
7 & Very, very strongly & 8 & \\
8 & Extremely & 9 & $($ from -8 to 0$)$ \\
$\{-i\}$ & Reciprocals/opposites of the above & (from $1 / 9$ to 1$)$ & \\
\hline
\end{tabular}

least squares (PLS) method and least penalty squares (LPS) method.

The PLS method derives the utility vector through the following model:

$$
\begin{array}{ll}
\min & \Delta=\sum_{i=1}^{n} \sum_{j=i+1}^{n}\left(b_{i j}-\left(v_{i}-v_{j}\right)\right)^{2}, \\
\text { s.t. } & \sum_{i=1}^{n} v_{i}=n \kappa, \\
& v_{i} \geq 0, \quad i=1,2, \ldots, n .
\end{array}
$$

Yuen [22] pointed out that the closed form solution of model (5) is the Row Average plus the normal Utility (RAU) which can be expressed as

$$
\begin{aligned}
& \operatorname{RAU}(B, \kappa) \\
& \quad=\left[v_{i}: v_{i}=\left(\frac{1}{n} \sum_{j=1}^{n} b_{i j}\right)+\kappa, \forall i=1,2, \ldots, n\right] .
\end{aligned}
$$

If there exists $v_{i}<0, \kappa$ can be increased such that $v_{i} \geq 0$.

LPS can be seen as the weighted version of PLS in which the weight is the penalty factor $\beta$. The utility vector can be derived by the following model:

$$
\begin{array}{ll}
\min & \widehat{\Delta}=\sum_{i=1}^{n} \sum_{j=i+1}^{n} \beta_{i j}\left(b_{i j}-v_{i}-v_{j}\right)^{2}, \\
\text { s.t. } & \sum_{i=1}^{n} v_{i}=n \kappa, \\
& v_{i} \geq 0, \quad i=1,2, \ldots, n, \\
& \beta_{i j} \quad \\
& = \begin{cases}\beta_{1}, & \left(v_{i}>v_{j} \wedge b_{i j}>0\right) \text { or }\left(v_{i}<v_{j} \wedge b_{i j}<0\right), \\
\beta_{2}, & \left(v_{i}=v_{j} \wedge b_{i j} \neq 0\right) \text { or }\left(v_{i} \neq v_{j} \wedge b_{i j}=0\right), \\
\beta_{3}, \quad \text { otherwise, } & \\
1 & =\beta_{1} \leq \beta_{2} \leq \beta_{3} .\end{cases}
\end{array}
$$

After obtaining the utility vector $V=\left\{v_{1}, v_{2}, \ldots, v_{n}\right\}$, the normalized priority vector can be obtained easily by

$$
W=\left\{w_{i} \mid w_{i}=\frac{v_{i}}{n \kappa}, i=1,2, \ldots, n\right\}
$$

MIP is the same as the aggregation process traditionally mentioned in MADM and DVP is the decision process based on the aggregated global utility of each alternative.

2.2. BWM. BWM is a vector based MADM method which requires fewer comparisons than the matrix based method such as AHP. Here we briefly describe the steps of BWM.

Step 1. Determine a set of decision criteria.

Step 2. Determine the best criterion $g_{B}$ and worst criterion $g_{W}$.

As is mentioned in [23], the decision-maker identifies the best and the worst criteria in general in this step. And if more than one criterion is considered to be the best or the worst, one can be chosen arbitrarily. No comparison is made at this stage.

Step 3. Execute pairwise comparisons between the best criterion and the other criteria. The result of the comparisons is the best-to-others vector denoted as

$$
A_{B}=\left(a_{B 1}, a_{B 2}, \ldots, a_{B n}\right)
$$

Step 4. Execute pairwise comparisons between the other criteria and the worst criterion. The result of the comparisons is the others-to-worst vector denoted as

$$
A_{W}=\left(a_{1 W}, a_{2 W}, \ldots, a_{n W}\right) .
$$


Step 5. Derive the weight vector $\left(w_{1}^{*}, w_{2}^{*}, \ldots, w_{n}^{*}\right)$. The weight vector can be obtained by solving the following problem:

$$
\begin{array}{ll}
\min & \xi, \\
\text { s.t. } & \left|\frac{w_{B}}{w_{i}}-a_{B i}\right| \leq \xi, \quad i=1,2, \ldots, n, \\
& \left|\frac{w_{i}}{w_{W}}-a_{i W}\right| \leq \xi, \quad i=1,2, \ldots, n, \\
& \sum_{i=1}^{n} w_{i}=1, \\
& w_{i} \leq 0, \quad i=1,2, \ldots, n .
\end{array}
$$

In (11), $w_{B}$ is the weight of the best criterion; $w_{W}$ is the weight of the worst criterion. Rezaei [24] pointed out that the solution of problem (11) is not unique when there are more than three criteria and the comparison vectors are not completely consistent and he proposed another linear model to derive the weight vector.

$$
\begin{array}{ll}
\min & \xi \\
\text { s.t. } & \left|w_{B}-a_{B i} w_{i}\right| \leq \xi, \quad i=1,2, \ldots, n, \\
& \left|w_{i}-a_{i W} w_{W}\right| \leq \xi, \quad i=1,2, \ldots, n, \\
& \sum_{i=1}^{n} w_{i}=1, \\
& w_{i} \leq 0, \quad i=1,2, \ldots, n .
\end{array}
$$

Let $\xi^{*}$ be the solution of problem (12); the consistency ratio of the pairwise comparisons in BWM is defined as

$$
\text { Consistency Ratio }=\frac{\xi^{*}}{\text { Consistency Index }} .
$$

Consistency index in (13) is the maximal possible value of $\xi$. Table 3 shows the consistency index for different values of $a_{B W}$ which is the preference of the best criterion over the worst criterion.

\section{Cognitive Best Worst Method}

In this section, we elaborate the proposed Cognitive Best Worst Method (CBWM) which can be seen as interval ratio based BWM. The basic process of CBWM is the same as BWM, so we focus on the differences caused by introducing interval ratio to represent pairwise difference.

We still use $A_{B}=\left(a_{B 1}, a_{B 2}, \ldots, a_{B n}\right)$ and $A_{W}=\left(a_{1 W}, a_{2 W}\right.$, $\left.\ldots, a_{n W}\right)$ to denote the best-to-others and others-to-worst vectors, while each element in the two vectors is expressed by interval scale as in Table 2 . The best and the worst criteria should be determined by the decision-maker in the same way as mentioned in BWM. Let $g_{B}$ be the best criterion and $g_{W}$ be the worst criterion; then $a_{B i}$ denotes the difference between $g_{B}$ and $g_{i}$, and $a_{i W}$ denotes the difference between $g_{i}$ and $g_{W}$.
It is notable that all $a_{B i}$ and $a_{i W}$ are nonnegative. As $a_{B W}$ is the difference between the best criterion and the worst criterion, we can get that $a_{B W}$ is the maximal element in both $A_{B}$ and $A_{W}$, and $a_{B W} \leq \kappa(\kappa$ is the normal utility and $\max (\bar{X})=\kappa)$.

Proposition 1. The pairwise comparison vectors $A_{B}$ and $A_{W}$ are perfectly accordant if

$$
\begin{aligned}
a_{B i}-a_{B j} & =a_{j W}-a_{i W}, \quad \forall i, j \in\{1,2, \ldots, n\}, \\
\text { or } a_{B i}+a_{i W} & =a_{B W}, \quad \forall i \in\{1,2, \ldots, n\} .
\end{aligned}
$$

Theorem 2. Two conditions (14) and (15) in Proposition 1 are equivalent.

Proof. Deduce (15) from (14). From (14) we can get $a_{B i}+a_{i W}=$ $a_{B j}+a_{j W}$ for all $i, j \in\{1,2, \ldots, n\}$; then by setting $j=B$ or $j=W$, we can get $a_{B i}+a_{i W}=a_{B B}+a_{B W}=a_{B W}+a_{W W}=a_{B W}$ for all $i \in\{1,2, \ldots, n\}$. Deduce (14) from (15). From (15) we can get $a_{B i}+a_{i W}=a_{B j}+a_{j W}=a_{B W}$ for all $i, j \in\{1,2, \ldots, n\}$. Then it is trivial to get (14).

3.1. Consistency Index. Based on Proposition 1, we define the consistency index $(\mathrm{CI})$ to measure the deviation of the pairwise comparison vectors $A_{B}$ and $A_{W}$ from consistency.

$$
\mathrm{CI}=\sqrt{\frac{1}{n} \sum_{i=1}^{n}\left(\frac{a_{B i}+a_{i W}-a_{B W}}{\kappa}\right)^{2}} .
$$

It is easy to verify that $\mathrm{CI} \geq 0$ and $\mathrm{CI}=0$ if and only if $A_{B}$ and $A_{W}$ are perfectly accordant.

Now we discuss the relation of CI in (16) and AI in (4). The basic ideas of CI and AI are the same, for they both measure the average deviation of each element in the pairwise comparison matrix (vector) from consistency. The values of $\mathrm{CI}$ and $\mathrm{AI}$ are comparable for the same MADM problem. We define the random consistency index $\mathrm{CI}_{R}$ as the mathematical expectation of CI when all the values except $a_{B W}$ in $A_{B}$ and $A_{W}$ are selected randomly and independently from the set $\left\{0,1, \ldots, a_{B W}\right\}$.

Theorem 3. Consider the following:

$$
\mathrm{CI}_{R}=\sqrt{\frac{n-2}{n} \frac{a_{B W}^{2}+2 a_{B W}}{6 \kappa^{2}}} .
$$

Proof. Let $\Delta_{i}=\left(\left(a_{B i}+a_{i W}-a_{B W}\right) / \kappa\right)^{2}$. When the $i$ th criterion is the best or the worst one, the value of $\Delta_{i}$ will be zero; otherwise the value of $\Delta_{i}$ will be in the closed interval [ 0 , $\left.\left(a_{B W} / \kappa\right)^{2}\right]$ and the corresponding probability distribution is given in Table 4 . It is easy to verify that $\sum_{k=0}^{a_{B W}} p\left(\Delta_{i}=(k / \kappa)^{2}\right)=$ 1. 
TABLE 3: Consistency index (CI) table of BWM.

\begin{tabular}{llcccccccc}
\hline$a_{B W}$ & 1 & 2 & 3 & 4 & 5 & 6 & 7 & 8 & 9 \\
\hline $\mathrm{CI}$ & 0 & 0.44 & 1.00 & 1.63 & 2.30 & 3.00 & 3.73 & 4.47 & 5.23 \\
\hline
\end{tabular}

TABLE 4: The probability distribution of $\Delta_{i}$.

\begin{tabular}{lllllll}
\hline$\Delta_{i}$ & 0 & $\frac{1}{\kappa^{2}}$ & $\ldots$ & $\frac{k^{2}}{\kappa^{2}}$ & $\ldots$ & $\frac{a_{B W}^{2}}{\kappa^{2}}$ \\
\hline$p\left(\Delta_{i}\right)$ & $\frac{a_{B W}+1}{\left(a_{B W}+1\right)^{2}}$ & $\frac{2 a_{B W}}{\left(a_{B W}+1\right)^{2}}$ & $\ldots$ & $\frac{2\left(a_{B W}+1-k\right)}{\left(a_{B W}+1\right)^{2}}$ & $\ldots$ & $\frac{2}{\left(a_{B W}+1\right)^{2}}$ \\
\hline
\end{tabular}

According to the probability distribution of $\Delta_{i}$, we can get the mathematical expectation of $\Delta_{i}$.

$$
\begin{aligned}
E\left(\Delta_{i}\right)=\sum_{k=1}^{a_{B W}} \frac{k^{2}}{\kappa^{2}} \frac{2\left(a_{B W}+1-k\right)}{\left(a_{B W}+1\right)^{2}} & \frac{2}{\kappa^{2}\left(a_{B W}+1\right)^{2}}\left[\sum_{k=1}^{a_{B W}}\left(a_{B W}+1\right) k^{2}-\sum_{k=1}^{a_{B W}} k^{3}\right] \\
= & \frac{2}{\kappa^{2}\left(a_{B W}+1\right)^{2}}\left[\frac{a_{B W}\left(a_{B W}+1\right)^{2}\left(2 a_{B W}+1\right)}{6}\right. \\
- & \left.\frac{a_{B W}^{2}\left(a_{B W}+1\right)^{2}}{4}\right]=\frac{a_{B W}^{2}+2 a_{B W}}{6 \kappa^{2}} .
\end{aligned}
$$

The values corresponding to different criteria in the two vectors $A_{B}$ and $A_{W}$ are independent, so we can get

$$
\mathrm{CI}_{R}=E\left(\sqrt{\frac{1}{n} \sum_{i=1}^{n} \Delta_{i}}\right)=\sqrt{\frac{n-2}{n} \frac{a_{B W}^{2}+2 a_{B W}}{6 \kappa^{2}}}
$$

For $\mathrm{AI}$, we define random accordance index $\mathrm{AI}_{R}$ as the mathematical expectation of AI when all the elements of the pairwise opposite matrix are selected from the set $\{-\kappa, \ldots,-1,0,1, \ldots, \kappa\}$ randomly and independently under the constraint that $b_{i j}=-b_{j i}$. To get the value of $\mathrm{AI}_{R}$, we run the simulation which generates the POM in the way as just described 10000 times and calculate AI each time; then we take the average of the 10000 values as the estimate of $\mathrm{AI}_{R}$.

Table 5 shows the values of $\mathrm{CI}_{R}$ and $\mathrm{AI}_{R}$ with different $n$ and $\kappa$ when $a_{B W}=\kappa$. From Table 5 we can clearly see that $\mathrm{AI}_{R}>\mathrm{CI}_{R}$ for the given $n$ and $\kappa$ which means that CBWM is more likely to get a more consistent pairwise comparison result than $\mathrm{P}-\mathrm{CNP}$. The reason of this phenomenon is that the elements in vectors of CBWM are all nonnegative while the elements of the POM may be either positive or negative, which leads to the fact that the maximal value of $\left(\left(a_{B i}+a_{i W}-\right.\right.$ $\left.\left.a_{B W}\right) / \kappa\right)^{2}$ is 1 and the maximal value of $\left(\left(b_{i k}+b_{k j}-b_{i j}\right) / \kappa\right)^{2}$ is 9.

The above analysis is valid under the circumstance that the decision-maker is able to appropriately determine the best and the worst criteria. Comparing the best and the worst objects to the others matches the habit and process of human cognition and then is likely to get more consistent results than comparing each pair of the objects. However, if the decisionmaker is not able to determine the best and the worst criteria, the above analysis is of no sense and CBWM method is not suitable for the problem.

Another advantage of CBWM is that it is easy to check the consistency and identify the cause of inconsistency. The examination and identification can be done intuitively with the help of Table 6. The first and the second rows are the values of $A_{B}$ and $A_{W}$, respectively. We can sum the elements in rows 1 and 2 and compare the results to $a_{B W}$. If $a_{B i}+a_{i W}$ is too different from $a_{B W}$, we should revise $A_{B}$ and $A_{W}$ to get more consistent vectors.

\subsection{Derivation of Utility Vector}

Theorem 4. When the comparison vectors $A_{B}$ and $A_{W}$ are completely consistent, the utility vector $V=\left(v_{1}, v_{2}, \ldots, v_{n}\right)$ can be derived by

$$
\begin{aligned}
v_{i} & =\frac{1}{n} \sum_{j=1}^{n} a_{B j}+\kappa-a_{B i}, \quad \forall i \in\{1,2, \ldots, n\}, \\
\text { or } v_{i} & =\kappa-\frac{1}{n} \sum_{j=1}^{n} a_{j W}+a_{i W}, \quad \forall i \in\{1,2, \ldots, n\} .
\end{aligned}
$$

Proof. When $A_{B}$ and $A_{W}$ are completely consistent, we can get $a_{B j}=v_{B}-v_{j}$ and $a_{j W}=v_{j}-v_{W}$ for all $j \in\{1,2, \ldots, n\}$. $\sum_{j=1}^{n} a_{j W}=n v_{B}-\sum_{j=1}^{n} v_{j}=n\left(v_{B}-\kappa\right)$, and $(1 / n) \sum_{j=1}^{n} a_{B j}+\kappa-$ $a_{B i}=v_{B}-\left(v_{B}-v_{i}\right)=v_{i}$; (20) holds. $\sum_{j=1}^{n} a_{j W}=\sum_{j=1}^{n} v_{j}-n v_{W}=$ $n\left(\kappa-v_{W}\right)$, and $\kappa-(1 / n) \sum_{j=1}^{n} a_{j W}+a_{i W}=v_{W}+\left(v_{i}-v_{W}\right)=v_{i}$; (21) holds.

When the comparison vectors $A_{B}$ and $A_{W}$ are not fully consistent, we propose two methods to derive the utility vector: the minimization of the maximal deviation method (MMD) and the minimization of total deviation method (MTD).

In MMD, the optimal utility vector $V=\left(v_{1}, v_{2}, \ldots, v_{n}\right)$ is the one where, for each pair of $v_{B}-v_{i}$ and $v_{i}-v_{W}$, we have $v_{B}-$ $v_{i}=a_{B i}$ and $v_{i}-v_{W}=a_{i W}$. To satisfy these conditions, we find a solution where the maximal absolute differences $\left|v_{B}-v_{i}-a_{B i}\right|$ and $\left|v_{i}-v_{W}-a_{i W}\right|$ for all $i \in\{1,2, \ldots, n\}$ are minimized. The 
TABLE 5: $\mathrm{CI}_{R}$ and $\mathrm{AI}_{R}$ with different $n$ and $\kappa$.

\begin{tabular}{llccccccccc}
\hline$n$ & & 3 & 4 & 5 & 6 & 7 & 8 & 9 & 10 \\
\hline \multirow{2}{*}{$\kappa=5$} & $\mathrm{CI}_{R}$ & 0.279 & 0.342 & 0.374 & 0.394 & 0.408 & 0.418 & 0.426 & 0.432 & 0.437 \\
& $\mathrm{AI}_{R}$ & 0.341 & 0.520 & 0.629 & 0.703 & 0.756 & 0.797 & 0.827 & 0.852 & 0.873 \\
$\kappa=6$ & $\mathrm{CI}_{R}$ & 0.272 & 0.333 & 0.365 & 0.385 & 0.398 & 0.408 & 0.416 & 0.422 & 0.426 \\
& $\mathrm{AI}_{R}$ & 0.337 & 0.512 & 0.621 & 0.694 & 0.746 & 0.785 & 0.816 & 0.841 & 0.861 \\
$\kappa=7$ & $\mathrm{CI}_{R}$ & 0.267 & 0.327 & 0.359 & 0.378 & 0.391 & 0.401 & 0.408 & 0.414 & 0.419 \\
& $\mathrm{AI}_{R}$ & 0.335 & 0.508 & 0.615 & 0.686 & 0.738 & 0.777 & 0.807 & 0.831 & 0.852 \\
$\kappa=8$ & $\mathrm{CI}_{R}$ & 0.264 & 0.323 & 0.354 & 0.373 & 0.386 & 0.295 & 0.403 & 0.408 & 0.412 \\
& $\mathrm{AI}_{R}$ & 0.330 & 0.504 & 0.609 & 0.681 & 0.731 & 0.770 & 0.801 & 0.825 & 0.846 \\
$\kappa=9$ & $\mathrm{CI}_{R}$ & 0.261 & 0.319 & 0.350 & 0.369 & 0.381 & 0.391 & 0.398 & 0.404 & 0.408 \\
& $\mathrm{AI}_{R}$ & 0.330 & 0.501 & 0.605 & 0.677 & 0.727 & 0.766 & 0.795 & 0.820 & 0.840 \\
\hline
\end{tabular}

TABLE 6: Consistency check (CC) table.

\begin{tabular}{|c|c|c|c|c|c|c|c|}
\hline & $g_{1}$ & $\cdots$ & $g_{B}$ & $\cdots$ & $g_{W}$ & $\cdots$ & $g_{n}$ \\
\hline$A_{B}$ & $a_{B 1}$ & $\cdots$ & 0 & $\cdots$ & $a_{B W}$ & $\cdots$ & $a_{B n}$ \\
\hline$A_{W}$ & $a_{1 W}$ & $\cdots$ & $a_{B W}$ & $\cdots$ & 0 & $\cdots$ & $a_{n W}$ \\
\hline$a_{B i}+a_{i W}$ & $a_{B 1}+a_{1 W}$ & $\cdots$ & $0+a_{B W}$ & $\cdots$ & $a_{B W}+0$ & $\cdots$ & $a_{n W}+a_{n W}$ \\
\hline CC & $=?$ & & $=?$ & & $=?$ & & $=?$ \\
\hline$a_{B W}$ & $a_{B W}$ & & $a_{B W}$ & & $a_{B W}$ & & $a_{B W}$ \\
\hline
\end{tabular}

optimal utility vector can be obtained by solving the following problem:

$$
\begin{array}{ll}
\min & \max _{i}\left\{\left|v_{B}-v_{i}-a_{B i}\right|,\left|v_{i}-v_{W}-a_{i W}\right|\right\}, \\
\text { s.t. } & \sum_{i=1}^{n} v_{i}=n \kappa, \\
& v_{i} \geq 0, \quad i=1,2, \ldots, n .
\end{array}
$$

Problem (22) can be transferred to the following linear programming problem:

$$
\begin{array}{ll}
\min & \xi \\
\text { s.t. } & \left|v_{B}-v_{i}-a_{B i}\right| \leq \xi, \quad i=1,2, \ldots, n, \\
& \left|v_{i}-v_{W}-a_{i W}\right| \leq \xi, \quad i=1,2, \ldots, n, \\
& \sum_{i=1}^{n} v_{i}=n \kappa \\
& v_{i} \geq 0, \quad i=1,2, \ldots, n .
\end{array}
$$

In MTD, the optimal utility vector should be the one where the total deviation $\sum_{i=1}^{n}\left(\left(v_{B}-v_{i}-a_{B i}\right)^{2}+\left(v_{i}-v_{W}-a_{i W}\right)^{2}\right)$ is minimized. It is natural to get the following model:

$$
\begin{array}{ll}
\min & \sum_{i=1}^{n}\left(\left(v_{B}-v_{i}-a_{B i}\right)^{2}+\left(v_{i}-v_{W}-a_{i W}\right)^{2}\right), \\
\text { s.t. } & \sum_{i=1}^{n} v_{i}=n \kappa, \\
& v_{i} \geq 0, \quad i=1,2, \ldots, n .
\end{array}
$$

Analogous to [22], we can get the closed form solution of model (24) as follows:

$$
\begin{aligned}
v_{i}= & \frac{v_{B}+v_{W}+a_{i W}-a_{B i}}{2}, \quad i \neq B, i \neq W, \\
v_{B}= & \kappa+\frac{n+1}{n(n+2)} \sum_{i=1}^{n} a_{B i}-\frac{1}{n(n+2)} \sum_{i=1}^{n} a_{i W} \\
& +\frac{1}{n+2} a_{B W}, \\
v_{W}= & \kappa+\frac{1}{n(n+2)} \sum_{i=1}^{n} a_{B i}-\frac{n+1}{n(n+2)} \sum_{i=1}^{n} a_{i W} \\
& -\frac{1}{n+2} a_{B W} .
\end{aligned}
$$

Also, if there exists $v_{i}<0, \kappa$ can be increased such that $v_{i} \geq 0$.

Solving problem (23) or problem (24), the optimal utility vector $\left(v_{1}^{*}, v_{2}^{*}, \ldots, v_{n}^{*}\right)$ is obtained. By normalizing $\left(v_{1}^{*}, v_{2}^{*}, \ldots, v_{n}^{*}\right)$, we can get the weight vector $W^{*}=\left(v_{1}^{*} / n \kappa\right.$, $\left.v_{2}^{*} / n \kappa, \ldots, v_{n}^{*} / n \kappa\right)$.

\subsection{Numerical Example}

3.3.1. Example 1. Supposing, in a MADM problem, there are four criteria $\left\{g_{1}, g_{2}, g_{3}, g_{4}\right\}$, among which $g_{1}$ is the best criterion and $g_{2}$ is the worst criterion. The comparison vectors provided by the experts are $A_{B}=(0,8,5,6)$ and $A_{W}=$ $(8,0,3,2)$ with $\kappa=8$. The aim is to get the weight vector which expresses the relative importance of each criterion. We first calculate the consistency index by (16) and we can get $\mathrm{CI}=0$. As $A_{B}$ and $A_{W}$ are fully consistent, we can use (20) or 
(21) to get the utility vector. The utility vector derived from (20) is $V=(12.74,4.75,7.75,6.75)$ and the corresponding weight vector is $W=(0.398,0.148,0.242,0.212)$.

3.3.2. Example 2. Here we revise Example 1 in [23] using CBWM to illustrate the CBWM steps discussed above. The example involves selecting a transport mode for a company. The criteria determined by the company are as follows: $g_{1}$ : load flexibility, $g_{2}$ : accessibility, and $g_{3}$ : cost. The company identifies that $g_{3}$ is the best criterion and $g_{1}$ is the worst criterion, and the original comparison vectors in ratio scale are $A_{B}=(8,2,1)$ and $A_{W}=(1,5,8)$. In interval scale, the corresponding comparison vectors will be $A_{B}=(7,1,0)$ and $A_{W}=(0,4,7)$ with $\kappa=8$.

Firstly, we calculate the consistency index by (16) and we can get $\mathrm{CI}=0.144$. To get the weight vector, we substitute the data into model (23) and model (24) and solve the two problems.

By MMD, we can get the utility vector $V=(4.33,9.0$, $10.67)$ and the weight vector $W=(0.181,0.375,0.444)$.

By MTD, we can get the utility vector $V=(4.2,9.0,10.8)$ and the weight vector $W=(0.175,0.374,0.451)$.

\section{Illustrations}

In this section, we compare the proposed MADM method CBWM to AHP, P-CNP, and BWM on a real world MADM problem.

4.1. Problem Description. Here the transport company selection problem discussed by $[7,35]$ is revised by AHP, P-CNP, BWM, and CBWM methods. A company needs to select a freight transportation company from four alternatives (T1, T2, T3, and T4) to deliver its goods. The criteria considered are transportation costs (TC), defective rate (DR), tardiness rate (TR), flexibility (F), and documentation ability (DA).

Six pairwise reciprocal matrices are shown in Table 7 . The top left matrix is the comparisons between criteria and the other five matrices are comparisons between alternatives with respect to different criteria. Saaty's consistency ratio is given under each matrix.

Table 8 shows six pairwise opposite matrices $(\kappa=8)$ under interval scale corresponding to the six matrices in Table 7. The accordance index (AI) defined in (4) is given under each matrix.

Tables 9 and 10 show the pairwise comparison vectors under ratio and interval scale, respectively. These vectors are extracted from the corresponding matrices. Best ${ }^{\#}$ represents the index of the best criterion (alternative) and Worst ${ }^{\#}$ represents the index of the worst criterion (alternative). It is worth noting that, on criterion TC, the alternatives T1 and T3 are equal and are both the worst. Rezaei [23] pointed out that if more than one criterion is considered to be the best or the worst, one can be chosen arbitrarily. Here we choose T1 as the worst with respect to TC.

The comparison matrices on criteria DR and DA are completely consistent in Table 8 while it is not the case in Table 7 . The phenomenon indicates the consistent cognition for pairwise comparison in a matrix can be distorted as inconsistent by ratio scale [7]. It is also the same when replacing the comparison matrices with comparison vectors. We can see that the comparison vectors on criteria DR and DA are completely consistent in Table 10 while it is not the case in Table 9. It is also worth noting that the pairwise comparison vectors are always consistent when the corresponding matrices are consistent under whatever scales while the opposite is obviously not the case. As per the results above and the analysis of consistency index in Section 3.1, we can say that CBWM can get more consistent pairwise comparisons than AHP, BWM, and P-CNP.

4.2. Results of Different Methods. Tables 11-15 show the prioritization and aggregation results along with the ranking of the alternatives from the best to the worst. The results of AHP in Table 11 are obtained by the eigenvector methods. The results of BWM in Table 12 are obtained by solving linear model (12). The results of P-CNP in Table 13 are obtained by the PLS method in (5). The results of CBWM-MMD in Table 14 are obtained by the MMD method in (23). The results of CBWM-MTD in Table 15 are obtained by the MTD method in (24).

The results in [7] show that P-CNP performs better than AHP because P-CNP gets stable rankings with different prioritization methods while AHP get fluctuant rankings with different prioritization methods. From our results in Tables 11-15, we can see that the BWM obtains similar results to AHP with respect to both ranking and total utility, while the two prioritization methods of CBWM both achieve results very close to the P-CNP's results. We can say that CBWM acquires more reliable results and performs better than BWM in this case based on the conclusion in [7].

4.3. Comparisons with respect to Total Deviation. In order to measure the performance of different methods further, we employ total deviation (TD) [36] to make the comparison. The original definition of total deviation (TD) is the Euclidean distance between the ratios of weights $w_{i} / w_{j}$ and their pairwise comparison $\operatorname{PRM}(i, j)$.

$$
\mathrm{TD}_{\text {ratio }}=\sum_{i=1}^{n} \sum_{j=1}^{n}\left(\operatorname{PRM}(i, j)-\frac{w_{i}}{w_{j}}\right)^{2} .
$$

$\mathrm{PRM}$ is the pairwise reciprocal matrix using ratio scale; $w_{i}$ and $w_{j}$ are the weights of the $i$ th criterion and the $j$ th criterion, respectively.

For interval scale, we need to modify the definition of TD as

$$
\mathrm{TD}_{\text {interval }}=\sum_{i=1}^{n} \sum_{j=1}^{n}\left(\operatorname{POM}(i, j)-\left(v_{i}-v_{j}\right)\right)^{2}
$$

POM is the pairwise opposite matrix using interval scale; $v_{i}$ and $v_{j}$ are the utilities of the $i$ th criterion and the $j$ th criterion, respectively.

As the numbers of pairwise comparisons of P-CNP (AHP) and CBWM (CWM) are $n^{2}$ and $2 n$, respectively, it is 
TABle 7: Pairwise comparison matrices under ratio scale.

\begin{tabular}{|c|c|c|c|c|c|c|c|c|c|c|}
\hline Criteria & TC & DR & TR & $F$ & $\mathrm{DA}$ & TC & $\mathrm{T} 1$ & $\mathrm{~T} 2$ & T3 & $\mathrm{T} 4$ \\
\hline TC & 1 & 5 & 3 & 5 & 9 & $\mathrm{~T} 1$ & 1 & $1 / 3$ & 1 & $1 / 5$ \\
\hline DR & $1 / 5$ & 1 & $1 / 2$ & $1 / 2$ & 7 & $\mathrm{~T} 2$ & 3 & 1 & 3 & $1 / 2$ \\
\hline TR & $1 / 3$ & 2 & 1 & $1 / 2$ & 7 & T3 & 1 & $1 / 3$ & 1 & $1 / 5$ \\
\hline $\mathrm{F}$ & $1 / 5$ & 2 & 2 & 1 & 8 & $\mathrm{~T} 4$ & 5 & 2 & 5 & 1 \\
\hline DA & $1 / 9$ & $1 / 7$ & $1 / 7$ & $1 / 8$ & 1 & & & & & \\
\hline \multicolumn{6}{|c|}{$\mathrm{CR}=0.0776$} & \multicolumn{5}{|c|}{$\mathrm{CR}=0.0015$} \\
\hline $\mathrm{DR}$ & $\mathrm{T} 1$ & $\mathrm{~T} 2$ & T3 & $\mathrm{T} 4$ & & TR & $\mathrm{T} 1$ & $\mathrm{~T} 2$ & T3 & $\mathrm{T} 4$ \\
\hline $\mathrm{T} 1$ & 1 & 7 & 3 & 5 & & $\mathrm{~T} 1$ & 1 & $1 / 5$ & $1 / 5$ & 2 \\
\hline $\mathrm{T} 2$ & $1 / 7$ & 1 & $1 / 5$ & $1 / 3$ & & $\mathrm{~T} 2$ & 5 & 1 & $1 / 3$ & 7 \\
\hline T3 & $1 / 3$ & 5 & 1 & 3 & & T3 & 5 & 3 & 1 & 7 \\
\hline $\mathrm{T} 4$ & $1 / 5$ & 3 & $1 / 3$ & 1 & & $\mathrm{~T} 4$ & $1 / 2$ & $1 / 7$ & $1 / 7$ & 1 \\
\hline \multicolumn{6}{|c|}{$\mathrm{CR}=0.0433$} & \multicolumn{5}{|c|}{$\mathrm{CR}=0.0631$} \\
\hline $\mathrm{F}$ & $\mathrm{T} 1$ & $\mathrm{~T} 2$ & T3 & $\mathrm{T} 4$ & & $\mathrm{DA}$ & $\mathrm{T} 1$ & $\mathrm{~T} 2$ & $\mathrm{~T} 3$ & $\mathrm{~T} 4$ \\
\hline $\mathrm{T} 1$ & 1 & 5 & $1 / 3$ & 3 & & $\mathrm{~T} 1$ & 1 & $1 / 5$ & $1 / 3$ & $1 / 3$ \\
\hline $\mathrm{T} 2$ & $1 / 5$ & 1 & $1 / 7$ & $1 / 3$ & & $\mathrm{~T} 2$ & 5 & 1 & 3 & 3 \\
\hline T3 & 3 & 7 & 1 & 7 & & $\mathrm{~T} 3$ & 3 & $1 / 3$ & 1 & 1 \\
\hline $\mathrm{T} 4$ & $1 / 3$ & 3 & $1 / 7$ & 1 & & $\mathrm{~T} 4$ & 3 & $1 / 3$ & 1 & 1 \\
\hline \multicolumn{6}{|c|}{$\mathrm{CR}=0.0518$} & \multicolumn{5}{|c|}{$\mathrm{CR}=0.0161$} \\
\hline
\end{tabular}

TABle 8: Pairwise comparison matrices under interval scale.

\begin{tabular}{|c|c|c|c|c|c|c|c|c|c|c|}
\hline Criteria & TC & DR & TR & $\mathrm{F}$ & DA & $\mathrm{TC}$ & $\mathrm{T} 1$ & $\mathrm{~T} 2$ & T3 & $\mathrm{T} 4$ \\
\hline $\mathrm{TC}$ & 0 & 4 & 2 & 4 & 8 & $\mathrm{~T} 1$ & 0 & -2 & 0 & -4 \\
\hline DR & -4 & 0 & -1 & -1 & 6 & $\mathrm{~T} 2$ & 2 & 0 & 2 & -1 \\
\hline TR & -2 & 1 & 0 & -1 & 6 & T3 & 0 & -2 & 0 & -4 \\
\hline $\mathrm{F}$ & -4 & 1 & 1 & 0 & 7 & $\mathrm{~T} 4$ & 4 & 1 & 4 & 0 \\
\hline DA & -8 & -6 & -6 & -7 & 0 & & & & & \\
\hline \multicolumn{6}{|c|}{$\mathrm{AI}=0.1168$} & \multicolumn{5}{|c|}{$\mathrm{AI}=0.0423$} \\
\hline $\mathrm{DR}$ & $\mathrm{T} 1$ & $\mathrm{~T} 2$ & T3 & $\mathrm{T} 4$ & & TR & $\mathrm{T} 1$ & $\mathrm{~T} 2$ & T3 & $\mathrm{T} 4$ \\
\hline $\mathrm{T} 1$ & 0 & 6 & 2 & 4 & & $\mathrm{~T} 1$ & 0 & -4 & -4 & 1 \\
\hline $\mathrm{T} 2$ & -6 & 0 & -4 & -2 & & $\mathrm{~T} 2$ & 4 & 0 & -2 & 6 \\
\hline $\mathrm{T} 3$ & -2 & 4 & 0 & 2 & & T3 & 4 & 2 & 0 & 6 \\
\hline $\mathrm{T} 4$ & -4 & 2 & -2 & 0 & & $\mathrm{~T} 4$ & -1 & -6 & -6 & 0 \\
\hline \multicolumn{6}{|c|}{$\mathrm{AI}=0$} & \multicolumn{5}{|c|}{$\mathrm{AI}=0.1030$} \\
\hline $\mathrm{F}$ & $\mathrm{T} 1$ & $\mathrm{~T} 2$ & T3 & $\mathrm{T} 4$ & & DA & $\mathrm{T} 1$ & $\mathrm{~T} 2$ & T3 & $\mathrm{T} 4$ \\
\hline $\mathrm{T} 1$ & 0 & 4 & -2 & 2 & & $\mathrm{~T} 1$ & 0 & -4 & -2 & -2 \\
\hline $\mathrm{T} 2$ & -4 & 0 & -6 & -2 & & $\mathrm{~T} 2$ & 4 & 0 & 2 & 2 \\
\hline T3 & 2 & 6 & 0 & 6 & & T3 & 2 & -2 & 0 & 0 \\
\hline $\mathrm{T} 4$ & -2 & 2 & -6 & 0 & & $\mathrm{~T} 4$ & 2 & -2 & 0 & 0 \\
\hline \multicolumn{6}{|c|}{$\mathrm{AI}=0.0846$} & \multicolumn{5}{|c|}{$\mathrm{AI}=0$} \\
\hline
\end{tabular}

TABle 9: Pairwise comparison vectors under ratio scale.

\begin{tabular}{lcccc}
\hline & Best $^{\#}$ & $A_{B}$ & Worst $^{\#}$ & $A_{W}$ \\
\hline Criteria & 1 & $(1,5,3,5,9)$ & 5 & $(9,7,7,8,1)$ \\
TC & 4 & $(5,2,5,1)$ & 1 & $(1,3,1,5)$ \\
DR & 1 & $(1,7,3,5)$ & 2 & $(7,1,5,3)$ \\
TR & 3 & $(5,3,1,7)$ & 4 & $(2,7,7,1)$ \\
F & 3 & $(3,7,1,7)$ & 2 & $(5,1,7,3)$ \\
DA & 2 & $(5,1,3,3)$ & 1 & $(1,5,3,3)$ \\
\hline
\end{tabular}


TABLE 10: Pairwise comparison vectors under interval scale.

\begin{tabular}{lcccc}
\hline & Best $^{\#}$ & $A_{B}$ & Worst $^{\#}$ & $A_{W}$ \\
\hline Criteria & 1 & $(0,4,2,4,8)$ & 5 & $(8,6,6,7,0)$ \\
TC & 4 & $(4,1,4,0)$ & 1 & $(0,2,0,4)$ \\
DR & 1 & $(0,6,2,4)$ & 2 & $(6,0,4,2)$ \\
TR & 3 & $(4,2,0,6)$ & 4 & $(1,6,6,0)$ \\
F & 3 & $(2,6,0,6)$ & 2 & $(4,0,6,2)$ \\
DA & 2 & $(4,0,2,2)$ & 1 & $(0,4,2,2)$ \\
\hline
\end{tabular}

TABLE 11: Results of AHP.

\begin{tabular}{lcccccc}
\hline AHP & TC & DR & TR & F & DA & Result \\
\hline W & 0.513 & 0.108 & 0.156 & 0.195 & 0.027 & \\
\hline T1 & 0.099 & 0.565 & 0.087 & 0.249 & 0.078 & 0.176 \\
T2 & 0.284 & 0.055 & 0.312 & 0.054 & 0.522 & 0.225 \\
T3 & 0.099 & 0.262 & 0.549 & 0.592 & 0.200 & 0.286 \\
T4 & 0.518 & 0.118 & 0.053 & 0.105 & 0.200 & 0.313 \\
\hline
\end{tabular}

TABLE 12: Results of BWM.

\begin{tabular}{lccccccc}
\hline BWM & TC & DR & TR & F & DA & Result \\
\hline W & 0.488 & 0.130 & 0.216 & 0.130 & 0.036 & & \\
\hline T1 & 0.099 & 0.572 & 0.144 & 0.235 & 0.089 & 0.187 \\
T2 & 0.272 & 0.067 & 0.240 & 0.069 & 0.518 & 0.221 \\
T3 & 0.109 & 0.226 & 0.559 & 0.595 & 0.196 & 0.288 \\
T4 & 0.520 & 0.136 & 0.057 & 0.101 & 0.196 & 0.304 \\
\hline
\end{tabular}

TABLE 13: Results of P-CNP.

\begin{tabular}{lccccccc}
\hline P-CNP & TC & DR & TR & F & DA & Result & Rank \\
\hline W & 0.290 & 0.200 & 0.220 & 0.225 & 0.065 & 0.188 & 0.246 \\
T1 & 0.203 & 0.344 & 0.195 & 0.281 & 0.313 & 0.235 \\
T2 & 0.273 & 0.156 & 0.313 & 0.156 & 0.359 & 0.250 & 0.288 \\
T3 & 0.203 & 0.281 & 0.344 & 0.203 & 0.250 & 0.231 \\
T4 & 0.320 & 0.219 & 0.148 & & & 4 \\
\hline
\end{tabular}

TABLE 14: Results of CBWM-MMD.

\begin{tabular}{lccccccc}
\hline CBWM-MMD & TC & DR & TR & F & DA & Result & Rank \\
\hline W & 0.292 & 0.201 & 0.224 & 0.217 & 0.067 & & \\
\hline T1 & 0.204 & 0.344 & 0.199 & 0.284 & 0.188 & 0.247 \\
T2 & 0.277 & 0.156 & 0.309 & 0.155 & 0.313 & 0.236 \\
T3 & 0.199 & 0.281 & 0.348 & 0.364 & 0.250 & 0.288 & 3 \\
T4 & 0.319 & 0.219 & 0.145 & 0.197 & 0.250 & 0.229 \\
\hline
\end{tabular}

TABLE 15: Results of CBWM-MTD.

\begin{tabular}{lccccccc}
\hline CBWM-MTD & TC & DR & TR & F & DA & Result & Rank \\
\hline W & 0.286 & 0.203 & 0.228 & 0.215 & 0.069 & & \\
\hline T1 & 0.202 & 0.344 & 0.199 & 0.289 & 0.188 & 0.248 & 0.237 \\
T2 & 0.277 & 0.156 & 0.309 & 0.159 & 0.313 & 0.286 \\
T3 & 0.199 & 0.281 & 0.342 & 0.357 & 0.250 & 1 \\
T4 & 0.322 & 0.219 & 0.150 & 0.195 & 0.250 & 0.230 \\
\hline
\end{tabular}


TABLE 16: TDs of different methods.

\begin{tabular}{lccccc}
\hline Methods & AHP & BWM & P-CNP & CBWM-MMD & CBWM-MTD \\
\hline TD & 4.534 & 7.532 & 0.416 & 0.513 & 0.471 \\
\hline
\end{tabular}

unfair to judge the performances of these methods directly by TD. To make fair comparison, we employ the average TD which is the quotient of MV divided by the number of pairwise comparisons. So the definitions of TD for each method would be

$$
\begin{aligned}
& \mathrm{TD}_{\mathrm{AHP}}=\frac{1}{n^{2}} \sum_{i=1}^{n} \sum_{j=1}^{n}\left(\operatorname{PRM}(i, j)-\frac{w_{i}}{w_{j}}\right)^{2}, \\
& \mathrm{TD}_{\mathrm{P}-\mathrm{CNP}}=\frac{1}{n^{2}} \sum_{i=1}^{n} \sum_{j=1}^{n}\left(\operatorname{POM}(i, j)-\left(v_{i}-v_{j}\right)\right)^{2}, \\
& \mathrm{TD}_{\mathrm{BWM}}=\frac{1}{2 n} \sum_{i=1}^{n}\left(\left(a_{B i}-\frac{w_{B}}{w_{i}}\right)^{2}+\left(a_{i W}-\frac{w_{i}}{w_{W}}\right)^{2}\right),
\end{aligned}
$$

$\mathrm{TD}_{\mathrm{CBWM}}$

$$
=\frac{1}{2 n} \sum_{i=1}^{n}\left(\left(a_{B i}-\left(v_{B}-v_{i}\right)\right)^{2}+\left(a_{i W}-\left(v_{i}-v_{W}\right)\right)^{2}\right) .
$$

Table 16 shows the TDs of different methods when determining the relative importance of each criterion. From the table, we can see that the total deviations of ratio scale based methods are significantly bigger than interval scale based methods; that is to say, the proposed CBWM performs better than BWM. It is worth noting that the TD of PCNP is smaller than CBWM, but it does not mean that PCNP performs better than CBWM. The reason is that the accordance index of P-CNP is 0.1168 while the consistency index of CBWM is 0.2016 . According to the analysis of the relationship of CI and AI in Section 3.1, the phenomenon that $\mathrm{AI}<\mathrm{CI}$ is unusual.

\section{Conclusion and Future Research}

In this paper, a new interval scale and vector based pairwise comparison MADM method CBWM (Cognitive Best Worst Method) is proposed. CBWM combines the ideas of P-CNP and BWM method and shows better characteristics than the two methods. The theoretical and experimental analyses demonstrate the good performance of CBWM. The excellent features CBWM shows are summarized as follows:

(1) CBWM is vector based method and needs less pairwise comparisons than matrix based methods. The numbers of pairwise comparisons are $2 n-3$ and $n(n-1) / 2$ for CBWM and matrix based methods, respectively, where $n$ is the number of objects involved in the comparisons.

(2) CBWM can get more consistent pairwise comparison results due to the use of interval scale and the best worst comparison strategy.
(3) As the interval scale is more suitable for measuring cognitive paired comparisons, CBWM acquires more reliable decision results than ratio scale based methods.

It is worth noting that CBWM is only suitable for the problem in which the decision-maker is able to determine the best and the worst criteria.

The future research will focus on two aspects; one is to apply CBWM to more real world MADM problems, and the other is to extent CBWM to fuzzy or interval judgments to capture the uncertainty in the process of MADM.

\section{Conflicts of Interest}

The authors declare that they have no conflicts of interest.

\section{Acknowledgments}

This work was supported by the Natural Science Foundation of Jiangsu Province (Grants no. BK20150720).

\section{References}

[1] S. Greco, M. Ehrgott, and J. R. Figueira, Multiple Criteria Decision Analysis: State of the Art Surveys, International Series in Operations Research \& Management Science, Springer, New York, NY, USA, 2016.

[2] E. Jacquet-Lagreze and J. Siskos, "Assessing a set of additive utility functions for multicriteria decision-making, the UTA method," European Journal of Operational Research, vol. 10, no. 2, pp. 151-164, 1982.

[3] S. Greco, V. Mousseau, and R. Słowiński, “Ordinal regression revisited: multiple criteria ranking using a set of additive value functions," European Journal of Operational Research, vol. 191, no. 2, pp. 416-435, 2008.

[4] T. L. Saaty, "How to make a decision: the analytic hierarchy process," Interfaces, vol. 24, no. 6, pp. 19-43, 1994.

[5] T. L. Saaty, "Decision making-the Analytic Hierarchy and Network Processes (AHP/ANP)," Journal of Systems Science and Systems Engineering, vol. 13, no. 1, pp. 1-35, 2004.

[6] C. Bana e Costa and J.-C. Vansnick, "MACBETH—an interactive path towards the construction of cardinal value functions," International Transactions in Operational Research, vol. 1, no. 4, pp. 489-500, 1994.

[7] K. K. F. Yuen, "The primitive cognitive network process: comparisons with the analytic hierarchy process," International Journal of Information Technology \& Decision Making, vol. 10, no. 4, pp. 659-680, 2011.

[8] B. Roy, "The outranking approach and the foundations of ELECTRE methods," Theory and Decision, vol. 31, no. 1, pp. 4973, 1991. 
[9] J.-P. Brans, P. Vincke, and B. Mareschal, "How to select and how to rank projects: the PROMETHEE method," European Journal of Operational Research, vol. 24, no. 2, pp. 228-238, 1986.

[10] S. Greco, B. Matarazzo, and R. Slowinski, "Rought sets theory for multicriteria decision analysis," European Journal of Operational Research, vol. 129, no. 1, pp. 1-47, 2001.

[11] P. J. van Laarhoven and W. Pedrycz, "A fuzzy extension of Saaty's priority theory," Fuzzy Sets and Systems, vol. 11, no. 3, pp. 229241, 1983.

[12] K. K. F. Yuen, "Fuzzy cognitive network process: comparisons with fuzzy analytic hierarchy process in new product development strategy," IEEE Transactions on Fuzzy Systems, vol. 22, no. 3, pp. 597-610, 2014.

[13] J. R. Figueira, S. Greco, B. Roy, and R. Słowiński, "An overview of electre methods and their recent extensions," Journal of MultiCriteria Decision Analysis, vol. 20, no. 1-2, pp. 61-85, 2013.

[14] J. Wallenius, J. S. Dyer, P. C. Fishburn, R. E. Steuer, S. Zionts, and K. Deb, "Multiple criteria decision making, multiattribute utility theory: recent accomplishments and what lies ahead," Management Science, vol. 54, no. 7, pp. 1336-1349, 2008.

[15] M. Behzadian, R. B. Kazemzadeh, A. Albadvi, and M. Aghdasi, "PROMETHEE: a comprehensive literature review on methodologies and applications," European Journal of Operational Research, vol. 200, no. 1, pp. 198-215, 2010.

[16] N. Subramanian and R. Ramanathan, "A review of applications of Analytic Hierarchy Process in operations management," International Journal of Production Economics, vol. 138, no. 2, pp. 215-241, 2012.

[17] C. Kahraman, S. C. Onar, and B. Oztaysi, "Fuzzy multicriteria decision-making: a literature review," International Journal of Computational Intelligence Systems, vol. 8, no. 4, pp. 637-666, 2015.

[18] K. K. F. Yuen, "Analytic hierarchy prioritization process in the AHP application development: a prioritization operator selection approach," Applied Soft Computing, vol. 10, no. 4, pp. 975-989, 2010.

[19] L. L. Thurstone, "A law of comparative judgment," Psychological Review, vol. 34, no. 4, pp. 273-286, 1927.

[20] K. K. F. Yuen, Cognitive network process with fuzzy soft computing technique in collective decision aiding [Ph.D. thesis], Hong Kong Polytechnic University, Hong Kong, 2009.

[21] K. K. F. Yuen, "Pairwise opposite matrix and its cognitive prioritization operators: comparisons with pairwise reciprocal matrix and analytic prioritization operators," Journal of the Operational Research Society, vol. 63, no. 3, pp. 322-338, 2012.

[22] K. K. F. Yuen, "The primitive cognitive network process in healthcare and medical decision making: comparisons with the analytic hierarchy process," Applied Soft Computing, vol. 14, no. 1, pp. 109-119, 2014.

[23] J. Rezaei, "Best-worst multi-criteria decision-making method," Omega, vol. 53, pp. 49-57, 2015.

[24] J. Rezaei, "Best-worst multi-criteria decision-making method: some properties and a linear model," Omega, vol. 64, pp. 126130, 2016.

[25] K. K. F. Yuen, "Software-as-a-Service evaluation in cloud paradigm: primitive cognitive network process approach," in Proceedings of the 2nd IEEE International Conference on Signal Processing, Communications and Computing (ICSPCC '12), pp. 119-124, August 2012.

[26] C. Guan and K. K. F. Yuen, "Towards a hybrid approach of primitive cognitive network process and agglomerative hierarchical clustering for music recommendation," in Proceedings of the 11th International Conference on Heterogeneous Networking for Quality, Reliability, Security and Robustness (QSHINE '15), pp. 206-209, Taipei, Taiwan, 2015.

[27] V. Q. Chen and K. K. F. Yuen, “Towards a hybrid approach of Primitive Cognitive Network Process and Self-Organizing Map for computer product recommendation," in Proceedings of the International Conference on Intelligent Computing and Internet of Things (ICIT '15), pp. 9-12, January 2015.

[28] K. Kam, F. Yuen, and M. Yuen, "Department course selection problem: the primitive cognitive network process approach," in Proceedings of the IEEE International Conference on Teaching, Assessment and Learning for Engineering (TALE '12), pp. H1C15-H1C-17, August 2012.

[29] K. K. F. Yuen, "Towards an evaluation strategy for consumer electronic products using primitive cognitive network process," in Proceedings of the IEEE International Conference on Consumer Electronics-Taiwan (ICCE-TW'15), pp. 72-73, June 2015.

[30] K. K. F. Yuen and W. Wang, "Towards a ranking approach for sensor services using primitive cognitive network process in," in Proceedings of the IEEE 4th Annual International Conference on Cyber Technology in Automation, Control, and Intelligent Systems (CYBER '14), pp. 344-348, Hong Kong, 2014.

[31] J. Rezaei, T. Nispeling, J. Sarkis, and L. Tavasszy, "A supplier selection life cycle approach integrating traditional and environmental criteria using the best worst method," Journal of Cleaner Production, vol. 135, pp. 577-588, 2016.

[32] S. Sadaghiani, K. W. Ahmad, J. Rezaei, and L. Tavasszy, "Evaluation of external forces affecting supply chain sustainability in oil and gas industry using Best Worst Method," in Proceedings of the 1st Mediterranean Gas and Oil International Conference (MedGO '15), pp. 1-4, April 2015.

[33] J. Rezaei, J. Wang, and L. Tavasszy, "Linking supplier development to supplier segmentation using Best Worst Method," Expert Systems with Applications, vol. 42, no. 23, pp. 9152-9164, 2015.

[34] N. Salimi and J. Rezaei, "Measuring efficiency of universityindustry Ph.D. projects using best worst method," Scientometrics, vol. 109, no. 3, pp. 1911-1938, 2016.

[35] O. Kulak and C. Kahraman, "Fuzzy multi-attribute selection among transportation companies using axiomatic design and analytic hierarchy process," Information Sciences, vol. 170, no. 2-4, pp. 191-210, 2005.

[36] B. Golany and M. Kress, "A multicriteria evaluation of methods for obtaining weights from ratio-scale matrices," European Journal of Operational Research, vol. 69, no. 2, pp. 210-220, 1993. 


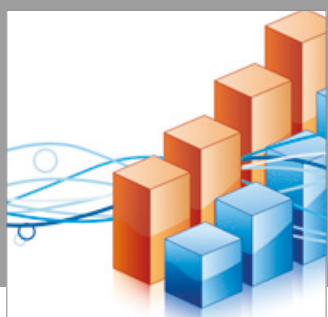

Advances in

Operations Research

vatersals

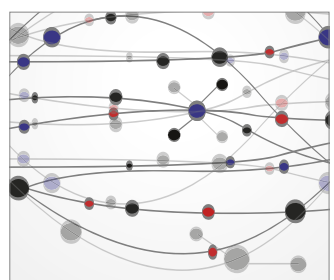

\section{The Scientific} World Journal
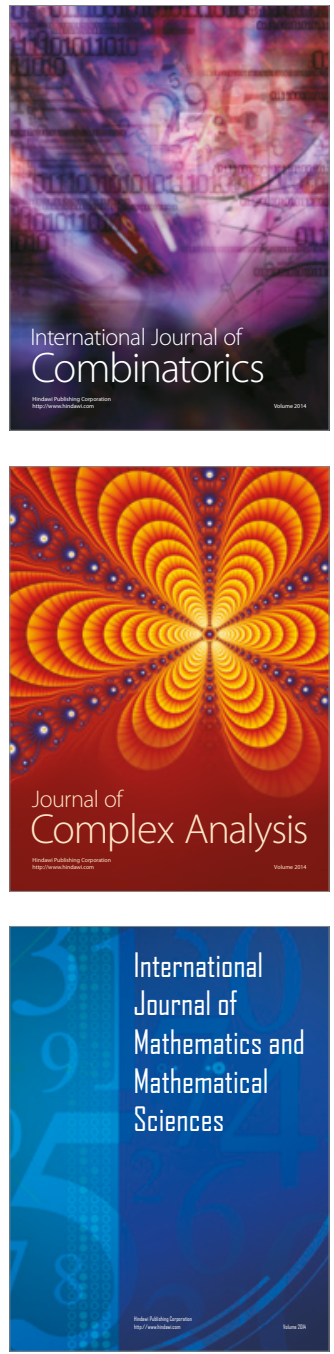
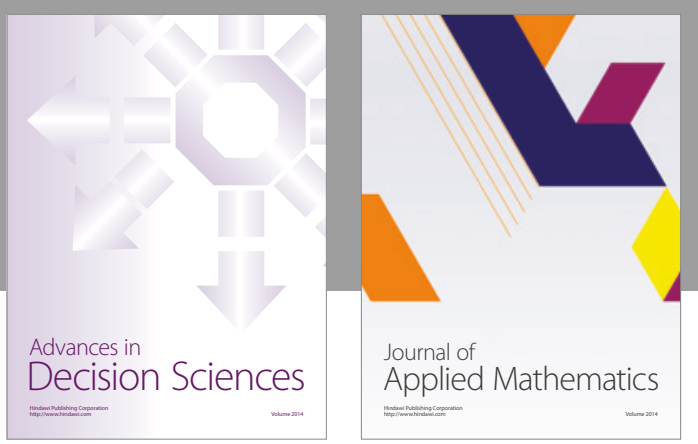

Algebra

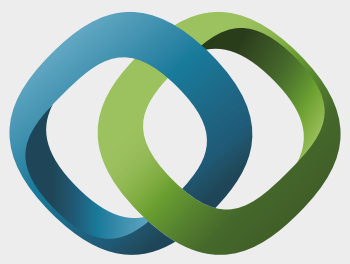

\section{Hindawi}

Submit your manuscripts at

https://www.hindawi.com
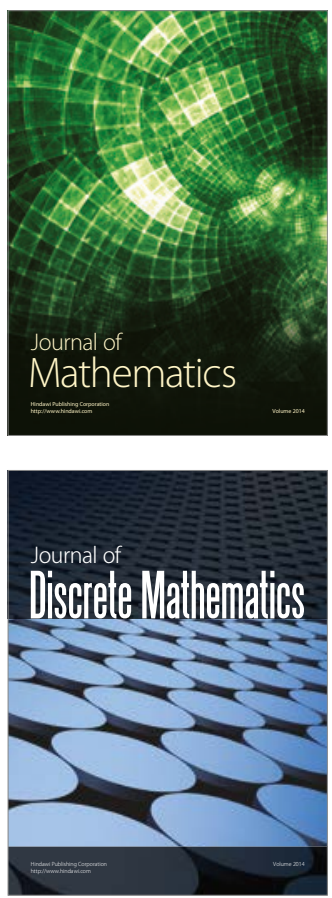

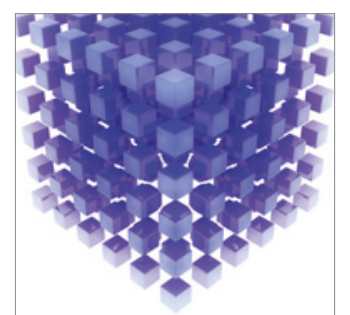

Mathematical Problems in Engineering
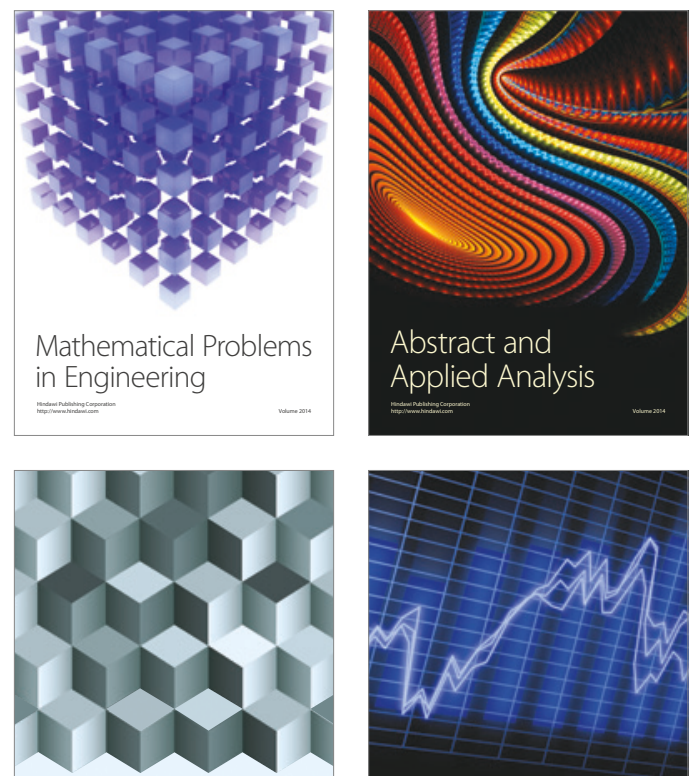

Journal of

Function Spaces

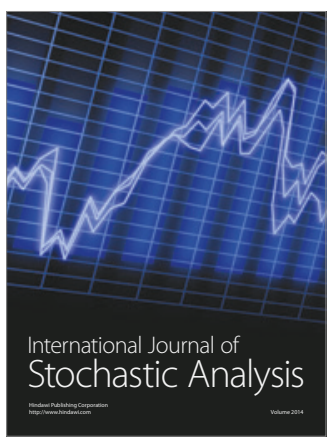

Probability and Statistics
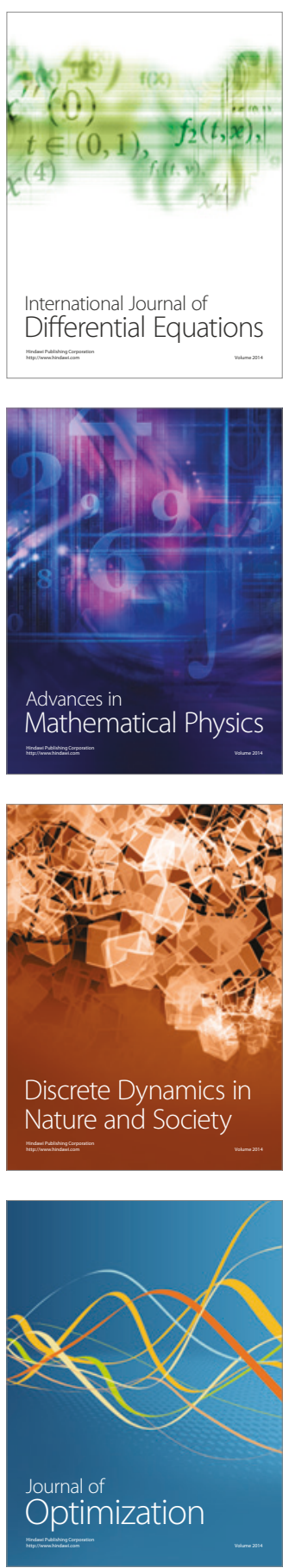